\title{
Effects of early experience on food preference in chicks'
}

GORDON M. BURGHARDT, University of Tennessee, Knoxville, Tennessee 37916

On the third day of life, four groups of eight chicks each were given either rape or canary seed, exclusively. The following day the chicks were given the seed they had not experienced the day previously. For eight days subsequently, the chicks were offered both seeds and the proportion by weight of each seed eaten was determined. Chicks initially fed canary seed showed a strong preference for canary when both seeds were first offered and the chicks initially fed rape seed showed no clear preference. As the days on both foods progressed, the preferences of both groups shifted toward rape seed, although group differences in favor of the food first fed existed throughout the testing period.

All animals must eat, but the relationship between what an animal eats early in life and his subsequent feeding behavior has been little studied. The considerable interest in sexual and parental imprinting in recent years has lead to speculation that similar early experience phenomena may occur in relation to food preferences (Thorpe, 1956; Hess, 1964). However, there is little experimental work with vertebrates that carefully controls the first and subsequent feeding of an organism. Kuo (1967) recently reported on a series of experiments on the "fixation of food habits" in young dogs, cats, and various birds. Although the procedures and results are not given in detail, his results clearly showed that six months on a given food item were sufficient to lead to a marked preference for it. Allen \& Littleford (1955) similarly showed the development of a preference for beef in diamondback terrapins (Malaclemys terrapin). Over a more restricted time span, Capretta \& Bronstein (1967) found that under certain conditions exposure to dyed mash for $25 \mathrm{~min}$ by $20-30$-h-old chicks had an effect on later food choice. However, in all these experiments the animals were tested for their preferences after exposure to only one food. Therefore, the effects of early experience cannot be separated from associative conditioning. This is also true of the work of Rabinowitch (1966) with gulls and Wood (1968) with snails. The latter, in fact, uses the term ingestive conditioning. Burghardt \& Hess (1966), on the other hand, found that not only did 12 feedings on a given food modify the newly-hatched snapping turtle's preference, but that an equivalent amount of experience on a second diet did not alter the original preference modification in a free-choice situation. Similarly, Burghardt (1967) showed that if inexperienced snapping turtles were given only one meal on each of two foods, the turtles later preferred the food first fed. In other words, a primacy effect was evident. This is clear evidence for the importance of the first over later foods.

Hess (1964) has presented evidence that supports the idea of the existence of a "food imprinting" type of phenomenon in chicks. He showed that food reinforcement on the third day of life led to an "irreversible" modification in the visual pecking preference, whereas reinforcement before or after this age had no lasting effect.

The present experiment is a preliminary study of the effects of primacy vs recency in the modification of actual food preferences in chicks. The first feeding was given on the third day of life, since Hess's experiment indicated that if a primacy effect in feeding experience did exist in chicks, it most likely would be found at that age. Groups of chicks were utilized to more closely approximate the natural situation in a social species, and to facilitate the onset of feeding behavior [Capretta \& Bronstein (1967) had to put their fingers in the food container to attract individual chicks or even place the chicks' heads into the food].

\section{METHOD}

All the experiments utilized Vantress broiler chicks in their third day of life. Two seeds were chosen that differed widely in appearance and, presumably, also in gustatory quality. The seeds were rape, a small round almost black seed, and canary, a yellow seed considerably longer than its diame ter and pointed at both ends. The chicks were maintained in groups in standard sized brooders ( $24 \times 36$ in.), each with a temperature gradient of 27 to $35 \mathrm{deg} C$. Water was always available and the room lights remained on 24 h a day.

In preliminary experiments, the seeds were presented to the chicks in glass finger bowls and the weight of seed eaten in $23 \mathrm{~h}$ was recorded. But it was soon discovered that the chicks scratched out of the bowls as much seed as they ate. These and other methodological problems led to the more precise experiments detailed here. However, one result worthy of mention was that seed eaten by 17 chicks with access to both seeds the first six days they were fed (age 3-8 days) averaged $61.5 \%$ for canary and $38.5 \%$ for rape over the six days with a range of 54 to $66 \%$ for canary and 34 to $46 \%$ for rape. Other pilot experiments gave results similar to those found in the experiments described below.

In the main series of experiments, the seeds were presented in one of the standard metal brooder troughs $(21 \mathrm{in}$.) that attach to the side of the brooder. The trough for each brooder was divided into four equal compartments with enough seed so that the chicks could peck at it without being able to jump into the seed and scratch it out. Only a negligible amount of seed was lost with this method. In addition, the high partitions prevented seed from entering adjacent compartments.

Four groups of eight newly-hatched chicks each were placed in to four brooders, in a rack, stacked one on top of the other. All four brooders, then, were oriented in the same direction with respect to light, temperature, and extraneous noise. The end troughs, opposite to the heating unit, held water. The troughs on one side were empty and wire retainers kept the chicks from going into them or escaping. The divided troughs were all placed on the same side of the brooders and the whole unit was moved so that the feeding area was evenly illuminated.

At the beginning of the experiment all 32 chicks were $54 \mathrm{~h}$ old (range: $52.56 \mathrm{~h}$ ). The topmost group and that second from the bottom were given canary seed as their first food on Day 3 , the other two groups were given rape seed. In the four compartments of each trough $75 \mathrm{~g}$ of seed were placed; in other words, each group of eight chicks was given $300 \mathrm{~g}$ of either rape or canary. The seed was first given at 6:00 PM. At 5:00 PM the following day, the troughs were removed and the remaining seed weighed. Then at 6:00 PM the troughs were replaced, with each group receiving the seed it had not experienced the preceding day. This $23-h$ feeding schedule was maintained throughout the duration of the experiment.

By Day 5, each chick had experienced both seeds but the order had varied. From Day 5 through Day 12 all chicks had their choice of both seeds. Different seeds were placed in adjacent compartments. Numbering the compartments from one end 1 to 4 , on Day 5 Compartments 1 and 3 contained canary for all groups and Compartments 2 and 4 contained rape. On Day 6, Compartments 2 and 4 held canary and 1 and 3 held rape. Day 7 was similar to Day 5, Day 8 to Day 6, and so on. In this way any cues that might influence the chicks to eat from a certain compartment were controlled and would affect all groups alike. Any seed which found its way into an adjacent compartment was removed with care before weighing.

\section{RESULTS}

In Fig. 1 is shown the percentage of canary seed eaten by each of the four groups during the period when both foods were offered. The most striking aspect of the graph is the wide divergence seen between the groups with respect to the seed first fed. The pilot study showed approximately a 3:2 preference for canary in chicks offered both foods. Giving canary on Day 3 led to about a 9:1 preference on Day 5, even though rape was given exclusively on Day 4. Similarly, the chicks fed rape on Day 3 showed a suppression of the canary preference to about the 1:1 level on Day 5, even though canary was fed exclusively on Day 4. Although the use of groups precludes a detailed statistical analysis, the fact that no 


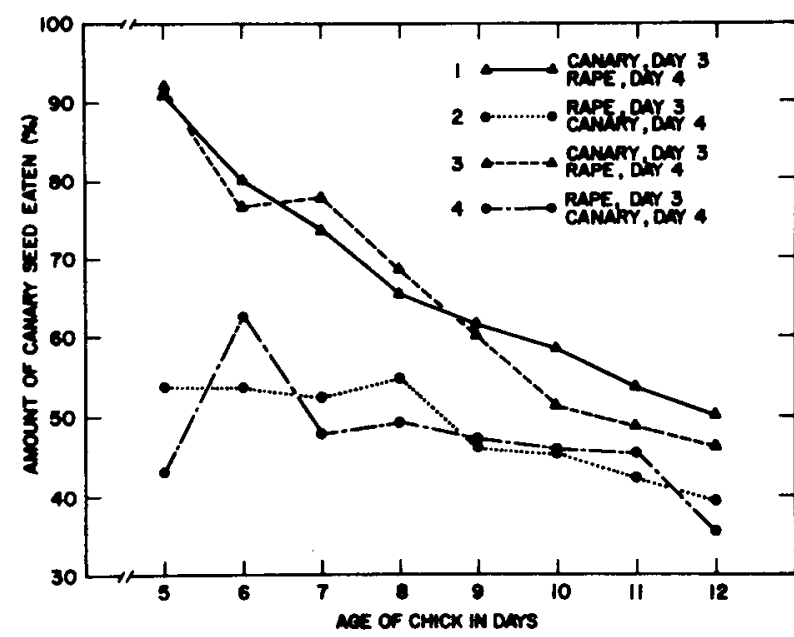

Fig. 1. Food choice by weight for chicks given both rape and canery seeds for eight days after being fed only one kind of seed on Day 3 and the other kind on Day 4. Eight chicks in each group.

overlap between two each of two differently treated groups occurred over eight days is in itself significant $(p<.01$, binomial test).

The next point to be made is that the difference between the groups decreased as the number of days on both foods increased. One would suspect that after Day 12 in this particular experiment the groups would begin to overlap. It is apparent, however, that this decreased divergence is also coupled with a shift from canary to rape preference for all groups. In any event, the prepotent effect of the seed first fed is clear.

All the chicks remained healthy throughout the experiment. However, they did not seem to gain as much weight or develop as quickly as normal chicks raised on mash. This was due, undoubtedly, to the fact that rape and canary seeds do not provide a sufficiently balanced diet.

\section{DISCUSSION}

The first food fed clearly had a primacy effect even though a different food was offered for an equivalent period of time. In this, the results are similar to those obtained in the snapping turtle (Burghardt \& Hess, 1966; Burghardt, 1967). The results are also compatible with those of Capretta \& Bronstein (1967) insofar as modification of the food preference was obtained in the same species. Of course, long-term studies would be desirable, as well as studies with chicks maintained singly.

The results are also compatible with Hess (1964) and the hypothesis that the third day is the critical period for "food imprinting" in chicks. However, to adequately test the critical period hypothesis, it would be necessary to pit the critical period against the primacy effect. This could be done by offering the two foods on the second and third days after hatching.

If no critical period is found, it is possible that a primacy effect could be demonstrated in older chickens if two novel food items were utilized, in a similar experiment. In other words, it might be argued that effects specific to early experience are not demonstrated in this or similar feeding experiments. While experiments with experienced Ss should certainly be attempted, it is difficult to envision positive results as clear as those with naive young. It would mean, for instance, that animals given successive exposures to foods $A B C D E$, would, after experiencing $A$ and $B$, prefer $A$. Then after experiencing Food C, prefer B, after experiencing D, prefer $\mathrm{C}$, and so on. This result should be obtained even if all five foods are utilized in each choice test throughout the experiment. The alternative hypothetical result suggested by the concept of the relative uniqueness of early feeding experiences is that forced alternation of different foods would lead to the disappearance of the primacy effect and its replacement by more potent, although perhaps transitory, preferences for the more recently experienced food.

Although the two seeds, undoubtedly, have differing nutritional values and metabolic effects, experience clearly plays an important role in food preferences of newly-hatched chicks. The tendency of all four groups to shift to rape seed as the days progressed indicated, however, that perhaps metabolic feedback can be important. This factor would appear to summate with the early-experience variable.

These findings warrant more detailed experiments on the ontogeny of seed preferences in chicks. The classical explanations of differential preferences stress structural factors such as bill shape and size (Lack, 1947). Lack even found subspecific differences in food habits among Darwin's finches. These differences were often associated with differences in beak size and shape. While the subspecies are probably now more adapted to their characteristic diets, it is possible that genetic morphological and behavioral differences associated with feeding arose from environmental and early experience processes. In any event, perceptual sign stimuli, metabolic factors, and early experience certainly cannot be ignored, especially within the range of foods which the animal is capable of ingesting with equal efficiency.

\section{REFERENCES}

ALLEN, J. F., \& LITTLEFORD, R. A. Observations of the feeding habits and growth of immature diamondback terrapins. Herpetologica, $1955,11,77-80$.

BURGHARDT, G. M. The primacy effect of the first feeding experience in the snapping turtle. Psychonomic Science, 1967, 7, 383-384.

BURGHARDT, G. M., \& HESS, E. H. Food imprinting in the snapping turtle, Chelydra serpentina Science, 1966, 151, 108-109.

CAPRETTA, P. J., \& BRONSTEIN, P. M. Effects of first food ingestion on later food preferences of chicks. Proceedings of the American Psychological Association 75th Annual Convention, 1967, 2, 109-110. HESS, E. H. Imprinting in birds. Science, 1964, 146, 1128-1139.

KUO, Z. Y. The dynamics of behavior development. New York: Random House, 1967

LACK, D. Darwin's finches. Cambridge: Cambridge University Press, 1947.

RABINOWITCH, V. The role of early experience in the development of food habits in gull chicks. Bulletin of the Ecological Society of America, 1966, 47, 144 (Abstract).

WOOD, L. Physiological and ecological aspects of prey selection by the marin gastropd, Urosalpinx cinerea (Prosobranchia: Muriciadae). Malacologia, 1968, 6, 267-320.

\section{NOTE}

1. Assisted by NIMH Grants MH 776, awarded to Eckhard $H$. Hess-whom I also thank for advice and MH 13375 and MH 15707 awarded to the author. The experiments were performed at the University of Chicago with the assistance of $\mathbf{L}$. S. Burghardt. 